\title{
Hydroxyalprazolam Measurement
}

National Cancer Institute

\section{Source}

National Cancer Institute. Hydroxyalprazolam Measurement. NCI Thesaurus. Code C147352.

The determination of the amount of hydroxyprazolam present in a sample. 